\title{
TOPOLOGICAL RELATIONSHIPS IN SPATIAL TESSELLATIONS
}

\author{
VIOLA WEISS, ${ }^{*}$ Fachhochschule Jena \\ RICHARD COWAN, ${ }^{* *}$ University of Sydney
}

\begin{abstract}
Tessellations of $\mathbb{R}^{3}$ that use convex polyhedral cells to fill the space can be extremely complicated. This is especially so for tessellations which are not 'facet-to-facet', that is, for those where the facets of a cell do not necessarily coincide with the facets of that cell's neighbours. Adjacency concepts between neighbouring cells (or between neighbouring cell elements) are not easily formulated when facets do not coincide. In this paper we make the first systematic study of these topological relationships when a tessellation of $\mathbb{R}^{3}$ is not facet-to-facet. The results derived can also be applied to the simpler facet-tofacet case. Our study deals with both random tessellations and deterministic 'tilings'. Some new theory for planar tessellations is also given.
\end{abstract}

Keywords: Random geometry; tessellation; tilings; packing of polyhedra; space filling; topology

2010 Mathematics Subject Classification: Primary 60D05; 05B45; 52C17

Secondary 60G55; 51M20; 52B10

\section{Introduction}

In this paper we study random stationary spatial tessellations, that is, random tessellations of the three-dimensional space $\mathbb{R}^{3}$ having statistical properties that are invariant under translation. We develop new mean-value formulae for various topological parameters in cases where the cells of the tessellation are convex polyhedra. The formulae generalize one of the identities for stationary planar tessellations presented by the second author [2], [3] in 1978. These identities, using notation based on his later papers (see [6] and [7]), are

$$
\begin{aligned}
\mu & =\frac{2 \theta}{\theta-2}, \\
\nu & =\frac{2(\theta-\phi)}{\theta-2} .
\end{aligned}
$$

Here $\mu$ indicates, for a typical cell, the expected number of edges (or vertices) of the tessellation on the cell's boundary. The entity $v$ is the expected number of sides (or corners) that the typical cell has. For a typical tessellation vertex, $\theta$ is the mean number of emanating edges. If a vertex has $j$ emanating edges, there are $j$ angles subtended by these edges at the vertex; if one of these angles is equal to $\pi$, the vertex is called a $\pi$-vertex. The parameter $\phi$ is defined as the proportion of vertices which are $\pi$-vertices. The words 'vertex' and 'edge' refer to the tessellation, whilst 'corner' and 'side' are words used for the 0 -faces and 1 -faces of the convex polygonal cells.

Received 12 March 2010; revision received 24 June 2011.

* Postal address: Fachhochschule Jena, D-07703 Jena, Germany. Email address: viola.weiss@fh-jena.de

** Postal address: School of Mathematics and Statistics, University of Sydney, NSW 2006, Australia.

Email address: rcowan@usyd.edu.au 


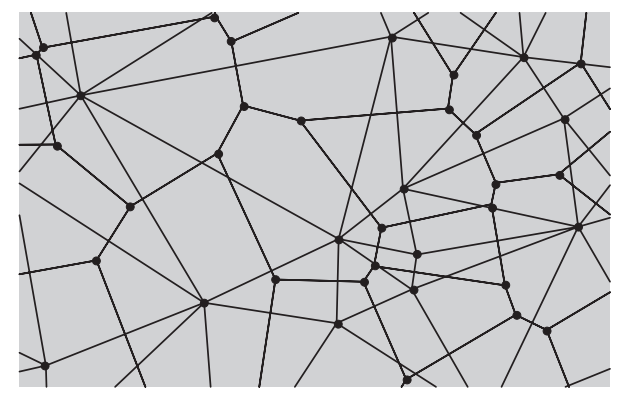

(a)

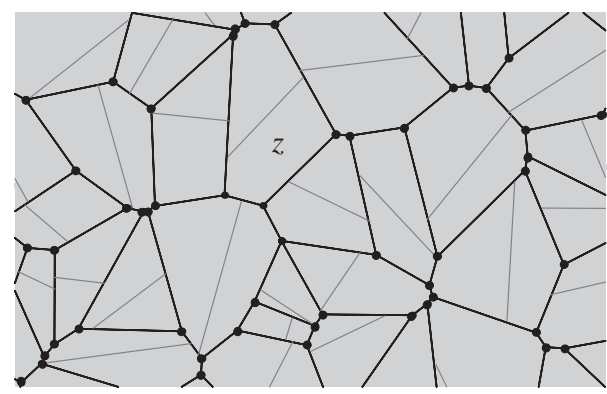

(b)

FIgure 1: (a) The planar tessellation formed as the superposition of a Poisson-Voronoi tessellation and its Poisson-Delaunay 'dual'. The tessellation is side-to-side-every edge is a 'side' of the two polygonal cells that it separates. Side-to-side tessellations have no $\pi$-vertices. (b) A Voronoi tessellation with each cell split by a random chord through the point used to generate that cell. These generating points are not shown; the original Voronoi vertices are shown with dots. Note that half of all vertices of the tessellation, those without dots, are $\pi$-vertices. The cell marked ' $z$ ' has five sides and seven edges on its boundary.

The two entities $\mu$ and $v$ are equal for a side-to-side tessellation (see Figure 1(a) for a definition of this terminology and an example), but differ if there exist $\pi$-vertices as in the example of Figure 1(b).

We illustrate (1) and (2) with four examples. In Figure 1(a), suppose that the Poisson point process that generates the tessellation has intensity $\rho$. Then the vertex intensities of the Delaunay and Voronoi components of the tessellation are $\rho$ and $2 \rho$, respectively. It is known (see [21]) that the intensity of the edge crossings of these two components is $4 \rho$. So $\theta=(6 \times \rho+3 \times 2 \rho+4 \times 4 \rho) / 7 \rho=4$. Since $\phi=0$, we can write $\mu=v=4$.

In Figure 1(b), both the Voronoi vertices and the new vertices created by the random chords have intensity $2 \rho$. So $\phi=\frac{1}{2}$. Each type is always of order three, so $\theta=3$. Therefore, $\mu=6$ and $v=5$.

The other two examples are based on Figure 2. In Figure 2(a), a realisation of the socalled stable with respect to iteration (STIT) model of Nagel and Weiss (see [14] and [22]) is shown. Here the tessellation within the window is constructed by the successive division of cells (in a particular manner) with independent random chords. This is one of a wide class of tessellations (recently systemised in [5]) which can be constructed in an iterative way. Many such constructions have similar characteristics to this picture, with all vertices being $\pi$-vertices and having order 3. Thus, $\phi=1$ and $\theta=3$; so $\mu=6$ and $v=4$. Although not constructed by an iterative division of cells, we note that one of the Arak class of models (see [1] and [17]) has similar parameters and so a similar calculation.

The tessellation in Figure 2(b) also comes from the iterative division of cells, but it starts with a triangular window and all random chords are constrained to create only triangular cells (see the caption of Figure 2, and [4] and [5]). Here, too, all vertices are $\pi$-vertices and all cells have three sides (so $\phi=1$ and $v=3$ ). Therefore, from (2), $3=2(\theta-1) /(\theta-2)$, which implies that $\theta=4$. From (1), $\mu=4$.

These examples illustrate that (2) is an essential adjunct to (1). In all the examples, we note that the fundamental parameters $\theta$ and $\phi$ satisfy the general constraints:

$$
0 \leq \phi \leq 1, \quad 3 \leq \theta \leq 6-2 \phi
$$




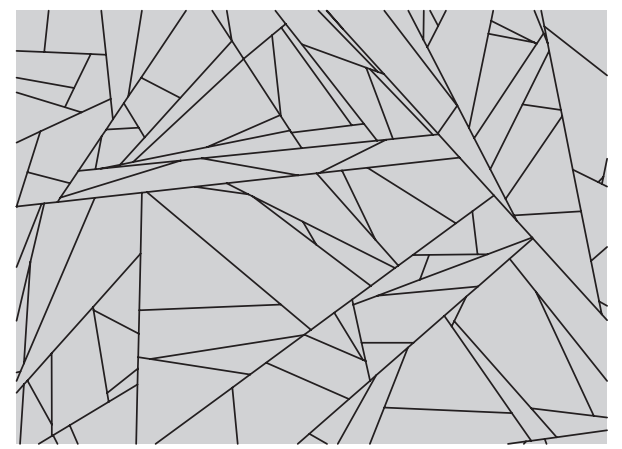

(a)

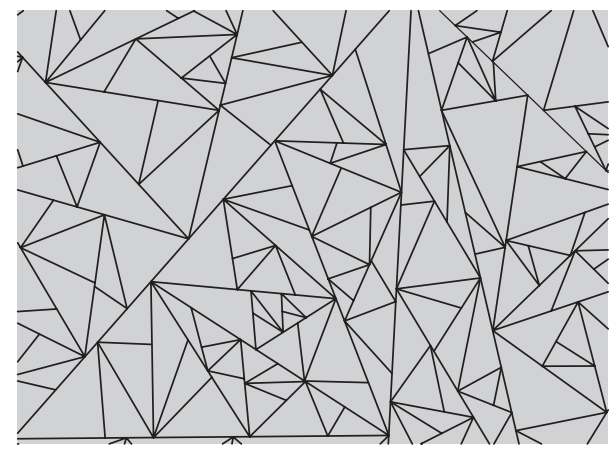

(b)

FIGURE 2: (a) A realisation of the STIT model within a rectangular viewing window. (b) This tessellation is constructed by the successive division of cells with independent random chords, commencing with a bounded triangular window which is the first cell in the process (the ancestor). The division chord is drawn from a randomly selected corner of the triangle to a random point on the opposite side, thereby creating two triangular daughter cells. This rule is applied iteratively, with dilation to keep the line intensity and mean cell size constant, to yield a tessellation comprising only triangular cells. If this construction converges to a stationary locally finite tessellation of $\mathbb{R}^{2}$ (an issue still under consideration in our studies), its realisation will look like the figure.

These constraints, which improve those derived by Kendall and Mecke [9], who made no use of $\phi$, apply to (1) and (2). The upper bound on $\theta$, which has not been reported before, follows from (2) and $v \geq 3$.

Strangely, few authors have paid any attention to the difference between edges and sides. Mecke [11], [13] developed (1), but not (2), and the standard text of Stoyan et al. [28, Sections 10.3 and 10.4] includes only (1) in a list of identities for stationary planar tessellations. In $\mathbb{R}^{3}$, there has been no discussion of the analogues of (2), although there have been generalisations of (1) (see [12], [25], and [28], together with the $d$-dimensional study of Møller [18]). Likewise, in the influential book of Schneider and Weil [26, Chapter 10] which discusses tessellations of $\mathbb{R}^{d}, d \geq 2$, only the facet-to-facet case is treated (though they use the equivalent face-to-face concept). These books and papers do not discuss the rather complicated issues that arise in three-dimensional tessellations which are not facet-to-facet.

Formula (2) is shown to be valid, for the planar case with nonconvex cells and curved edges, in [7], but the issue is not discussed in the studies of planar structures with curved cell boundaries by either Stoyan [27] or Miles [16]. Nor has there been reference to formulae like (2) in the studies of more general tessellations, those where the structure is a cell complex in $\mathbb{R}^{d}$ constructed with various systems of nonconvex cells (see [10], [30], and [31]). This is perhaps understandable as those cell-complex papers deal with purely topological structures not tainted by the geometric concepts of 'angles equal to $\pi$ '.

So there is a gap in the literature; our current paper fills this gap. It provides the first systematic study of the complications in $\mathbb{R}^{3}$ when a tessellation is not facet-to-facet, whilst also giving some new theory for the planar case.

\section{Notational style}

With some exceptions that we have cited, the studies mentioned above deal only with the primitive elements of the tessellation: in the planar case, the vertices, edges, and cells of the 
planar graph that the structure creates. In $\mathbb{R}^{3}$, the two-dimensional polygonal plates which separate the three-dimensional cells are also considered, along with vertices, edges, and cells. All of these entities are convex polytopes.

These primitive elements are sometimes referred to as 0-cells, 1-cells, 2-cells, and (in the spatial case) 3-cells, reflecting the dimension of the element-in keeping with cell-complex theory. This has led to notation such as $\mu_{i j}:=$ the expected number of $j$-cells adjacent to the typical $i$-cell and $\lambda_{i}:=$ the intensity of $i$-cells, by which is meant the intensity of the point process of centroids of $i$-cells. For example, $\mu_{01}$ is the expected number of edges 'adjacent to' (that is, 'emanating from') the typical vertex-Cowan's $\theta$ in the planar case.

We note that, for each class of objects in the realised tessellation, there is a point process formed from the objects' centroids. When the class comprises a primitive type of element, the point process is simple - that is, all centroids are distinct points of the Euclidean space, so there are no 'multiple points' of the point process. Later, when we consider other objects, the centroids will often coincide, forming a nonsimple point process with multiplicities. For example, in $\mathbb{R}^{2}$, the corners of cells will induce a point process with a multiplicity at each vertex of the tessellation, and the centroid of a cell's side will often coincide with the centroid of a neighbouring cell's side, giving a multiplicity of 2 . Indeed, the two sides in such cases will often coincide - they are the same geometric object, distinguished by the implicit link each has to a cell.

Because our study looks at many objects which have the same dimension, we are unable to rely solely on subscripts which convey the object's dimension. For example, in $\mathbb{R}^{2}$ the symbol $\mu_{21}$ would be ambiguous, meaning both the expected number of edges and the expected number of sides adjacent to a typical cell (because edges and sides are both of dimension 1).

Instead, we use letter subscripts for the primitive elements, namely $V, E, P$, and $Z$ for the object classes vertices, edges, plates, and cells (' $Z$ for Zellen' is used instead of ' $C$ for cell' because one of us has used ' $C$ for corner' in earlier work).

For objects which are the faces of primitives, e.g. the sides (1-faces) and corners ( 0 -faces) of polygonal primitives (such as cells in $\mathbb{R}^{2}$ or plates in $\mathbb{R}^{3}$ ), we retain some use of dimensional subscripts whilst conveying by letter the type of primitive element which 'owns' the face.

Definition 1. Let $X$ be a class of convex polytopes, each member of the class having dimension $i \leq 3$. Define $X_{j}, j<i$, as the class of objects which are $j$-dimensional faces ( $j$-faces) of some polytope belonging to $X$. Further nesting is allowed, so $\left(X_{j}\right)_{k}, k<j<i$, denotes the class of objects which are $k$-faces of a $j$-face of some object belonging to $X$. An object belonging to $X$ is often referred to as 'an $X$-type object' or 'an object of type $X$ '.

In $\mathbb{R}^{2}$ for example, an object of type $Z_{1}$ is a side of a cell, $Z_{0}$ denotes the objects which are corners of cells, and $E_{0}$ is the class of termini of edges. In $\mathbb{R}^{3}$, objects of types $P_{1}$ and $\left(Z_{2}\right)_{1}$ are respectively a side of a plate and a side of a facet (which is a 2-face of a cell). A 0 -face or 1-face of a three-dimensional polyhedral cell—called an apex or ridge, respectively-is in the class $Z_{0}$ or $Z_{1}$. A terminus of a ridge is type $\left(Z_{1}\right)_{0}$. Because of possible multiplicities, discussed above, some of these classes are multisets.

Remark 1. For some purposes, it may be necessary to emphasise the ownership of an object in $X_{j}$. For example, when considering a cell corner $z_{0} \in Z_{0}$, we might wish to distinguish this corner from the other cell corners at the same position. In that circumstance, we must place an ownership 'mark' on each element of $Z_{0}$, and deal with a product space $Z_{0}^{+}:=Z_{0} \times M$, where $M$ is the mark space comprising elements of $Z$. In this paper we have no need for this augmentation; Definition 1 is sufficient. 
The notation in Definition 1 appears complicated, but many simpler styles were tried and found inadequate. We shall assist verbally where appropriate, however, referring to an object's face as a facet, ridge, or apex for objects which are polyhedra, as a side or corner for polygons, and as a terminus for line segments.

Subsets of the class $X$ are denoted by $X[\cdot]$, with the term in the square brackets being a suitably chosen symbol introduced in an ad hoc manner. For example, in $\mathbb{R}^{2}$, the subclass of $\pi$-vertices is $V[\pi]$.

We now formalize the notation for 'object intensity' and the concept of 'adjacent objects'.

Definition 2. The intensity of objects belonging to class $X$ is denoted by $\lambda_{X}$.

Definition 3. An object $x \in X$ is said to be adjacent to an object $y \in Y$ if either $x \subseteq y$ or $y \subseteq x$. For any $x \in X$, the number of objects of type $Y$ adjacent to $x$ is denoted by $m_{Y}(x)$. For a random tessellation, we define $\mu_{X Y}$ as the expected value of $m_{Y}(x)$ when $x$ is the typical member of $X$. Formally, we write $\mu_{X Y}:=\mathrm{E}_{X}\left(m_{Y}(x)\right)=\int m_{Y}(x) \mathrm{P}_{X}(\mathrm{~d} x)$, where $\mathrm{E}_{X}$ denotes an expectation for the typical object of type $X$ (that is, defined with respect to the Palm measure $\mathrm{P}_{X}$; see [28]). Also, $\mu_{X Y}^{(2)}:=\mathrm{E}_{X}\left(m_{Y}(x)^{2}\right)$.

If $B(r)$ is the ball of radius $r$ centred at the origin, $\mu_{X Y}$ can also be defined formally as

$$
\mu_{X Y}:=\lim _{r \rightarrow \infty} \frac{\sum_{\{x \in X: \text { centroid of } x \text { is in } B(r)\}} m_{Y}(x)}{\text { number of objects of type } X \text { with centroid in } B(r)},
$$

when the limit shown is a constant. This is so for ergodic tessellations (see [2] and [3]) and for regular tilings with an infinitely repeated subunit of cells (as in [8]). This latter type can be made to fit our random stationary framework by locating the origin $O$ uniformly distributed within one copy of the repeating subunit. The limit in (4) is also a constant for many tessellations that have a 'tiling component', for example, those randomly constructed from a tiling or those mixed with or superimposed on a tiling.

When $X$ and $Y$ are both primitive-element classes, it has been shown in [10], [11], and [30] that

$$
\lambda_{X} \mu_{X Y}=\lambda_{Y} \mu_{Y X}
$$

and this identity also holds when either $X$ or $Y$ or both are classes of faces of primitives; Theorem 5.1 of [18] provides the proof of this extension. For example, when discussing sides of plates, $\lambda_{P_{1}} \mu_{P_{1} Z}=\lambda_{Z} \mu_{Z P_{1}}$.

We can express $\theta$ and $\phi$, symbols used by Cowan in planar tessellations, using the adjacency notation; $\theta=\mu_{V E}$ and $\phi=\mu_{V Z_{1}}$, the expected number of 'side interiors' adjacent to a typical vertex (where the interior of a side, or indeed of any object $x$ of lower dimension than the space of the tessellation, is defined using the relative topology on $x$ ). Also, $\stackrel{\circ}{X}$ denotes the class comprising the relative interiors of objects in class $X$ (and $\partial X$ denotes the class comprising boundaries, defined once more using the appropriate relative topology). Whilst we drop the usage of $\theta$ from this point, preferring $\mu_{V E}$, we retain $\phi$.

\section{Known results for the primitive elements}

For the primitive objects in a planar tessellation, Table 1 gives the known values of $\lambda_{X}$ and $\mu_{X Y}$. The table, based on the studies cited above, includes (1). 
TABLE 1: Results for the primitive elements in tessellations of $\mathbb{R}^{2}$. (a) $\lambda_{X}$ values for $X \in\{V, E, Z\}$. Note that $\lambda_{V}-\lambda_{E}+\lambda_{Z}=0$. (b) $\mu_{X Y}$ when both $X$ and $Y$ belong to $\{V, E, Z\}$. Note the validity of (5). We also note that $\lambda_{V[\pi]}=\phi \lambda_{V}$.

\begin{tabular}{|c|c|c|c|c|c|}
\hline \multicolumn{2}{|c|}{ (a) $\lambda_{X}$ values } & \multicolumn{4}{|c|}{ (b) $\mu_{X Y}$ values } \\
\hline \multirow{2}{*}{$X$} & \multirow{2}{*}{$\lambda_{X} / \lambda_{V}$} & \multirow{2}{*}{$X$} & \multicolumn{3}{|c|}{$Y$} \\
\hline & & & $V$ & $E$ & $Z$ \\
\hline Vertices $V$ & 1 & $V$ & 1 & $\mu_{V E}$ & $\mu_{V E}$ \\
\hline Edges $E$ & $\frac{1}{2} \mu_{V E}$ & E & 2 & 1 & 2 \\
\hline Cells $Z$ & $\frac{\mu_{V E}-2}{2}$ & $Z$ & $\frac{2 \mu_{V E}}{\mu_{V E}-2}$ & $\frac{2 \mu_{V E}}{\mu_{V E}-2}$ & 1 \\
\hline
\end{tabular}

TABLE 2: Results in $\mathbb{R}^{3}$ using the abbreviation $f$, defined in (6). (a) $\lambda_{X}$ when $X \in\{V, E, P, Z\}$. Note that $\lambda_{V}-\lambda_{E}+\lambda_{P}-\lambda_{Z}=0$. (b) Values of $\mu_{X Y}$ for primitive-element classes $X$ and $Y$. Note that $\mu_{V E}-\mu_{V P}+\mu_{V Z}=2$ and $\mu_{Z V}-\mu_{Z E}+\mu_{Z P}=2$.

\begin{tabular}{|c|c|c|c|c|c|c|}
\hline \multicolumn{2}{|c|}{ (a) $\lambda_{X}$ values } & \multicolumn{5}{|c|}{ (b) $\mu_{X Y}$ values } \\
\hline \multirow{2}{*}{$X$} & \multirow{2}{*}{$\lambda_{X} / \lambda_{V}$} & \multirow{2}{*}{$X$} & \multicolumn{4}{|c|}{$Y$} \\
\hline & & & $V$ & $E$ & $P$ & $Z$ \\
\hline Vertices $V$ & 1 & V & 1 & $\mu_{V E}$ & $\frac{1}{2} \mu_{V E} \mu_{E P}$ & $\frac{1}{2} f(2)$ \\
\hline Edges $E$ & $\frac{1}{2} \mu_{V E}$ & E & 2 & 1 & $\mu_{E P}$ & $\mu_{E P}$ \\
\hline Plates $P$ & $\frac{\mu_{V E} \mu_{E P}}{2 \mu_{P V}}$ & $P$ & $\mu_{P V}$ & $\mu_{P V}$ & 1 & 2 \\
\hline Cells $Z$ & $\frac{f\left(\mu_{P V}\right)}{2 \mu_{P V}}$ & $Z$ & $\frac{\mu_{P V} f(2)}{f\left(\mu_{P V}\right)}$ & $\frac{\mu_{V E} \mu_{E P} \mu_{P V}}{f\left(\mu_{P V}\right)}$ & $\frac{2 \mu_{V E} \mu_{E P}}{f\left(\mu_{P V}\right)}$ & 1 \\
\hline
\end{tabular}

Also known are the same entities for the primitive elements of spatial tessellations. In Table 2 we write these in terms of $\mu_{V E}, \mu_{E P}$, and $\mu_{P V}$, a trio with cyclic subscripts, and use

$$
f(x):=\mu_{V E} \mu_{E P}-x\left(\mu_{V E}-2\right)
$$

as a useful abbreviation.

\section{The faces of primitive elements: planar case}

We now calculate some results for faces of the primitive elements, dealing with the planar case first. To find $\lambda_{Z_{1}}$, the intensity of sides, we note that $\mu_{Z_{1} E}-\mu_{Z_{1} V}^{\circ}=1$. Therefore,

$$
\begin{aligned}
\lambda_{Z_{1}} & =\lambda_{Z_{1}} \mu_{Z_{1} E}-\lambda_{Z_{1}} \mu_{Z_{1} V}^{\circ} \\
& =\lambda_{E} \mu_{E Z_{1}}-\lambda_{V} \mu_{V Z_{1}} \\
& =2 \lambda_{E}-\phi \lambda_{V} \\
& =\left(\mu_{V E}-\phi\right) \lambda_{V},
\end{aligned}
$$

using identity (5), $\phi=\mu_{V Z_{1}}$ (each vertex being adjacent to either 0 or 1 side interiors), and the obvious $\mu_{E Z_{1}}=2$ (each edge being adjacent to two sides). 
Formula (7) leads on to (2) — with assistance from some new notation which generalizes Cowan's entity $v$.

Definition 4. Let $X$ be a class of convex polytopes, all members of which have dimension $i$. For $j<i$, we define $n_{j}(x)$ as the number of $j$-faces of a particular object $x \in X$. Define $v_{j}(X):=\mathrm{E}_{X}\left(n_{j}(x)\right)$, the expected number for the typical $X$-object.

The notation allows nesting; for example, $v_{k}\left(X_{j}\right)$ is the expected number of $k$-faces of the typical object of type $X_{j}(k<j<i$, where $i=\operatorname{dim} x$ for all $x \in X)$. An example with two levels of nesting occurs in spatial tessellations: $v_{0}\left(\left(Z_{2}\right)_{1}\right)$ is the expected number of termini of a typical 'side of a facet' of a cell.

Some examples of the definition in the fairly simple planar case are as follows.

- $v_{1}(Z)=$ the expected number of sides of the typical cell (Cowan's $v$ ). We can write $\lambda_{Z_{1}}=\lambda_{Z} v_{1}(Z)$. Therefore, using (7) and Table 1 ,

$$
v_{1}(Z)=\frac{\lambda_{Z_{1}}}{\lambda_{Z}}=\frac{2\left(\mu_{V E}-\phi\right)}{\mu_{V E}-2},
$$

providing a fresh proof of Cowan's $v$ (see (2)). Of course, $v_{0}(Z)=v_{1}(Z)$ and this leads to $\lambda_{Z_{0}}=\lambda_{Z} \nu_{0}(Z)=\left(\mu_{V E}-\phi\right) \lambda_{V}$.

- $v_{0}(E)=$ the mean number of edge termini of the typical edge. Trivially, $v_{0}(E)=2$, so $\lambda_{E_{0}}=\lambda_{E} v_{0}(E)=\mu_{V E} \lambda_{V}$.

- $v_{0}\left(Z_{1}\right)=$ the mean number of side termini of the typical side. Obviously, $v_{0}\left(Z_{1}\right)=2$, so $\lambda\left(Z_{1}\right)_{0}=\lambda_{Z_{1}} v_{0}\left(Z_{1}\right)=2\left(\mu_{V E}-\phi\right) \lambda_{V}$.

Table 3 summarizes the planar $\lambda$ - and $\nu$-results expressed in terms of $\mu_{V E}$ and $\phi$.

Table 4 gives various planar $\mu$-results, proved in Appendix A. Many of these results use the second moment, $\mu_{V E}^{(2)}$, and $\mu_{V[\pi] E}$, the expected number of edges adjacent to the typical $\pi$-vertex. The following function is a useful abbreviation:

$$
g(x) \begin{cases}:=\mu_{V E}^{(2)}-x \phi \mu_{V[\pi] E}, & \phi>0, \\ =\mu_{V E}^{(2)}, & \phi=0 .\end{cases}
$$

We conclude our material on planar tessellations by restating that our results summarised in Tables 1, 3, and 4 should be read with the constraints of (3) in mind.

\begin{tabular}{|c|c|c|c|c|}
\hline \multicolumn{2}{|c|}{$\lambda_{X}$ values } & \multicolumn{3}{|c|}{$v_{j}(X), j \leq 1$, values } \\
\hline$X$ & $\lambda_{X} / \lambda_{V}$ & $X$ & $v_{0}(X)$ & $v_{1}(X)$ \\
\hline Termini $E_{0}$ & $\mu_{V E}$ & Edges $E$ & 2 & - \\
\hline Corners $Z_{0}$ & $\mu_{V E}-\phi$ & Sides $Z_{1}$ & 2 & - \\
\hline \multirow{2}{*}{ Sides $Z_{1}$} & \multirow{2}{*}{$\mu_{V E}-\phi$} & \multirow{3}{*}{ Cells Z } & $2\left(\mu_{V E}-\phi\right)$ & $2\left(\mu_{V E}-\phi\right)$ \\
\hline & & & $\mu_{V E}-2$ & $\mu_{V E}-2$ \\
\hline Side termini $\left(Z_{1}\right)_{0}$ & $2\left(\mu_{V E}-\phi\right)$ & & & \\
\hline
\end{tabular}

TABLE 3: Results for faces of the primitive elements (and faces of faces) in planar tessellations. 
TABLE 4: Further $\mu_{X Y}$ values for the primitive-element classes $V, E$, and $Z$ in a planar tessellation, and, for those classes, $E_{0},\left(Z_{1}\right)_{0}, Z_{0}$, and $Z_{1}$, whose members are faces of (or faces of faces of) primitives.

These supplement those $\mu_{X Y}$ values in Table 2(b). The function $g$ is defined in (9).

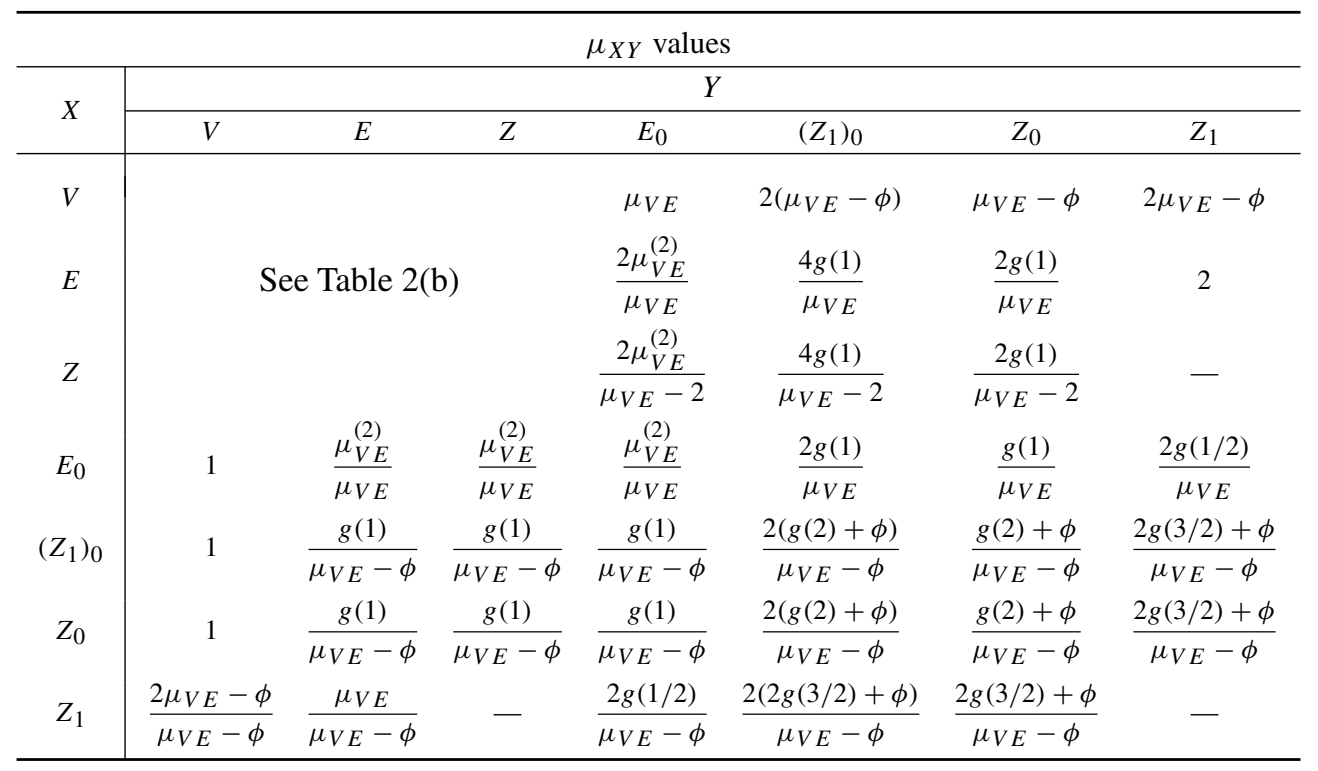

\section{Description of facets in a spatial tessellation}

The situation in $\mathbb{R}^{3}$ has much greater complexity, as seen in Figure 3, which shows a particular facet $z_{2}$ of a cell-plus some of the other cells that 'interact' with it. In Figure 3(a), we note that $z_{2}$ comprises eight polygonal plates. The interior of $z_{2}$ has considerable structure; it contains three vertices and 10 edge interiors of the tessellation. These numbers agree with the following Eulerian formula for any facet $z_{2} \in Z_{2}$ :

$$
m_{V}\left(\stackrel{\circ}{z}_{2}\right)-m_{E}\left(\stackrel{\circ}{z}_{2}\right)+m_{P}\left(z_{2}\right)=1 .
$$

Formula (10) follows from an application of Euler's planar-graph formula to yield

$$
m_{V}\left(z_{2}\right)-m_{E}\left(z_{2}\right)+m_{P}\left(z_{2}\right)=1,
$$

combined with $m_{V}\left(\partial z_{2}\right)=m_{E}\left(\partial z_{2}\right)$, for all $z_{2} \in Z_{2}$.

Along each of the 10 edge interiors mentioned above, the two coplanar plates of $z_{2}$ which meet along the edge make a dihedral angle equal to $\pi$ and, at the three vertices in $z_{2}$ 's interior, all emanating edges are contained in a closed hemisphere centred on the vertex.

Definition 5. An edge whose interior is contained in the interior of a facet is called a $\pi$-edge; the class of $\pi$-edges is $E[\pi]$. A vertex contained in the interior of a facet is called a hemivertex (and the class called $V[h]$ ). The proportion of $\pi$-edges in the tessellation is denoted by $\xi:=\mu_{E Z_{2}}^{\circ}$ and the proportion of hemi-vertices by $\kappa:=\mu_{V Z_{2}}^{\circ}$.

There are eight other cells positioned above $z_{2}$ which share a plate of this facet with the cell below $z_{2}$; two of these are shown in Figure 3(b) and we note that their facets extend beyond the shared plates. The triangular prism seen in Figure 3(c) packs between the two cells of 


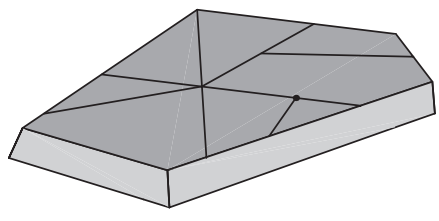

(a)

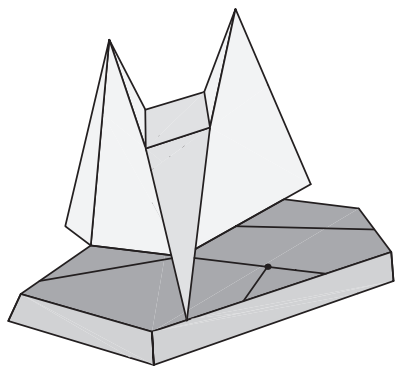

(c)

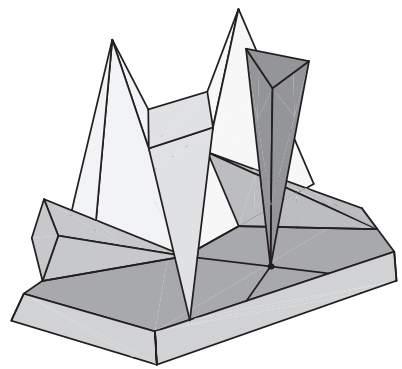

(e)

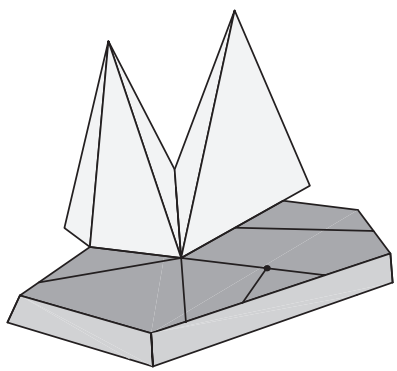

(b)

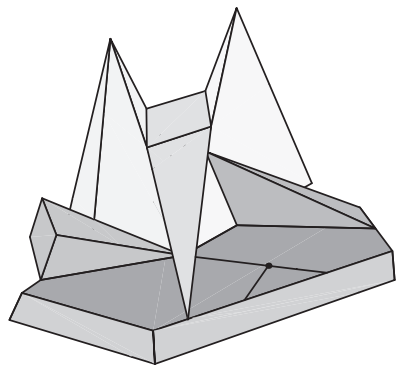

(d)

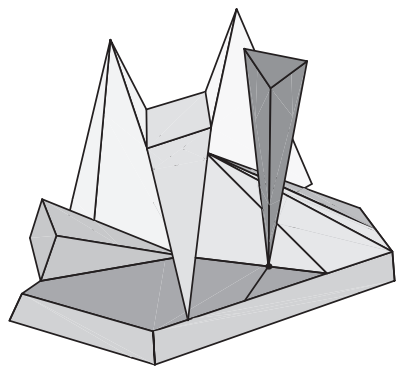

(f)

Figure 3: A cell facet $z_{2} \in Z_{2}$ and some neighbouring cells. The cell positioned below $z_{2}$ has $z_{2}$ as one of its facets.

Figure 3(b) and has one of its ridges lying along two collinear edges of our facet (seen in Figure 3(a)).

Two other 'above cells' which share a plate with the cell below are shown in Figure 3(d), whilst Figure 3(e) shows a cell (the tall dark one) whose only contact with our facet $z_{2}$ is via one of its apices; the apex coincides with an interior vertex of $z_{2}$. Yet another cell packs in behind the tall dark one in Figure 3(f), and there are other cells (not drawn) which fill the foreground.

From (10), we can derive $\lambda_{Z_{2}}$, the intensity of facets. We have

$$
\mu_{Z_{2} V}^{\circ}-\mu_{Z_{2} E}^{\circ}+\mu_{Z_{2} P}=1
$$


Therefore,

$$
\begin{aligned}
\lambda_{Z_{2}} & =\lambda_{Z_{2}} \mu_{Z_{2} V}^{\circ}-\lambda_{Z_{2}} \mu_{Z_{2} E}^{\circ}+\lambda_{Z_{2}} \mu_{Z_{2} P} \\
& \left.=\lambda_{V} \mu_{V Z_{2}}-\lambda_{E} \mu_{E Z_{2}}^{\circ \circ}+\lambda_{P} \mu_{P Z_{2}} \quad \text { (using (5) and } \lambda_{E}^{\circ}=\lambda_{E}\right) \\
& \left.=\kappa \lambda_{V}-\xi \lambda_{E}+2 \lambda_{P} \quad \text { (because } \mu_{P Z_{2}}=2\right) \\
& =\left(\kappa-\frac{1}{2} \xi \mu_{V E}+\frac{\mu_{V E} \mu_{E P}}{\mu_{P V}}\right) \lambda_{V} \quad \text { (from Table 2). }
\end{aligned}
$$

Furthermore, since $\lambda_{Z_{2}}$ can also be written as $\lambda_{Z} \nu_{2}(Z)$, we can derive $v_{2}(Z)$, the expected number of faces of the typical cell. We have

$$
\begin{aligned}
\nu_{2}(Z) & =\frac{\lambda_{Z_{2}}}{\lambda_{Z}} \\
& =\frac{2 \kappa \mu_{P V}-\xi \mu_{V E} \mu_{P V}+2 \mu_{V E} \mu_{E P}}{f\left(\mu_{P V}\right)} \quad \text { (from (11) and Table 2) } \\
& =\mu_{Z P}-\mu_{P V} \frac{\xi \mu_{V E}-2 \kappa}{f\left(\mu_{P V}\right)} .
\end{aligned}
$$

This is our first formula which is analogous to Cowan's planar formula (8). Other analogous formulae for $v_{1}(Z)$ and $v_{0}(Z)$ follow in the next section.

\section{Description of ridges in a spatial tessellation}

Figure 4(a) shows two facets of a cell, each containing some edges. These edges create three vertices on the ridge which are common to the two facets.

There may, however, be other vertices in the ridge interior. For example, another cell may have an apex on the ridge, or it might have one of its ridges intersecting with the ridge, creating one or two vertices additional to those shown in the figure.

So we must recognise that a vertex of the tessellation may lie in a ridge interior (or indeed in many ridge interiors). This is true even for hemi-vertices. The expected number of ridge

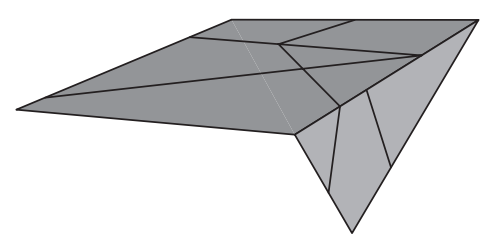

(a)

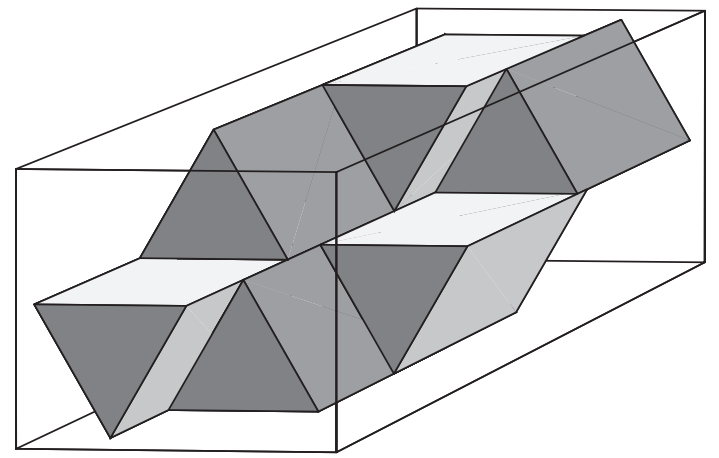

(b)

FIGURE 4: (a) Two facets of a cell and the ridge which is their intersection. (b) Six cells from a tessellation formed by congruent triangular prisms. The vertical axis is the $z$-axis, whilst the horizontal axes are the $x$-axis (pointing right) and the $y$-axis (pointing to the back). 
interiors adjacent to a typical vertex, $\psi:=\mu_{V Z_{1}}$, turns out to be an important parameter of spatial tessellations.

Example 1. Consider Figure 4(b). Both triangular end facets of the prism are equilateral triangles of side length equal to 1 . These facets are parallel to each other and to the $x z$-plane. They are orthogonal to the other three facets (which are $1 \times L$ rectangles). The tessellation is made up of cells of this type. The cells are packed around a central axis which is orthogonal to the $x z$-plane (and so parallel to the horizontal $y$-axis), thereby forming an infinite length hexagonal rod. The rods pack to fill $\mathbb{R}^{3}$.

The positioning of cells is such that whenever the $x z$-plane cuts a cell, the distance between the cell's front triangular facet and the $x z$-plane is uniformly distributed in $(0, L)$, independently for each cell which is cut. (The origin $O$ is uniformly distributed within one such cell.) So triangular facets (which always comprise one whole plate) never share an edge. The edges of the triangles are $\pi$-edges; there is one of these $\pi$-edges lying against each rectangular facet, dividing the facet into two rectangular plates. Every vertex is adjacent to five ridge interiors, so $\psi=5$.

In order to find $v_{1}(Z)$, we note that

$$
\mu_{Z_{1} E}-\mu_{Z_{1} V}^{\circ}=1
$$

Therefore, using

$$
\lambda_{Z_{1}} \mu_{Z_{1} E}=\lambda_{E}\left(\mu_{E P}-\xi\right),
$$

together with Table $2,(5)$, and $\lambda_{Z_{1}}^{\circ}=\lambda_{Z_{1}}$,

$$
\begin{aligned}
\lambda_{Z_{1}} & =\lambda_{Z_{1}} \mu_{Z_{1} E}-\lambda_{Z_{1}}^{\circ} \mu_{Z_{1} V}^{\circ} \\
& =\lambda_{E}\left(\mu_{E P}-\xi\right)-\lambda_{V} \mu_{V Z_{1}} \\
& =\frac{1}{2}\left(\mu_{V E}\left(\mu_{E P}-\xi\right)-2 \psi\right) \lambda_{V} .
\end{aligned}
$$

Because $\lambda_{Z_{1}}$ also equals $\lambda_{Z} v_{1}(Z)$, Table 2 gives

$$
\begin{aligned}
v_{1}(Z) & =\frac{\lambda_{Z_{1}}}{\lambda_{Z}} \\
& =\frac{\mu_{P V}\left(\mu_{V E}\left(\mu_{E P}-\xi\right)-2 \psi\right)}{f\left(\mu_{P V}\right)} \\
& =\mu_{Z E}-\mu_{P V} \frac{\xi \mu_{V E}+2 \psi}{f\left(\mu_{P V}\right)} .
\end{aligned}
$$

So, from Euler's polyhedral formula, supplemented by (12), (15), and the information given in the caption of Table 2,

$$
\begin{aligned}
v_{0}(Z) & =v_{1}(Z)-v_{2}(Z)+2 \\
& =2 \mu_{P V} \frac{\mu_{V Z}-(\kappa+\psi)}{f\left(\mu_{P V}\right)} \\
& =\mu_{Z V}-\mu_{P V} \frac{2(\kappa+\psi)}{f\left(\mu_{P V}\right)} .
\end{aligned}
$$

Therefore,

$$
\lambda_{Z_{0}}=\lambda_{Z} v_{0}(Z)=\left(\mu_{V Z}-\kappa-\psi\right) \lambda_{V} .
$$


Every ridge is the side of two facets. So

$$
\begin{aligned}
\lambda_{\left(Z_{2}\right)_{1}} & =2 \lambda_{Z_{1}}, \\
\mu_{\left(Z_{2}\right)_{1} E} & =\mu_{Z_{1} E}=\frac{\lambda_{E}}{\lambda_{Z_{1}}}\left(\mu_{E P}-\xi\right) \quad(\text { from (13)). }
\end{aligned}
$$

Identity (18) yields formulae for $v_{1}\left(Z_{2}\right)$ and $v_{0}\left(Z_{2}\right)$, the expected numbers of sides and corners of the typical facet. Trivially, $\lambda_{Z_{2}} v_{1}\left(Z_{2}\right)=\lambda_{\left(Z_{2}\right)_{1}}$. Therefore, using (11) and (14),

$$
\nu_{1}\left(Z_{2}\right)=\frac{2 \lambda_{Z_{1}}}{\lambda_{Z_{2}}}=\frac{2 \mu_{P V}\left(\mu_{V E}\left(\mu_{E P}-\xi\right)-2 \psi\right)}{2 \mu_{V E} \mu_{E P}-\mu_{P V}\left(\xi \mu_{V E}-2 \kappa\right)} .
$$

Obviously, $v_{0}\left(Z_{2}\right)=v_{1}\left(Z_{2}\right)$.

\section{Sides and corners of the typical plate}

Following the methods employed above, we note that

$$
\mu_{P_{1} E}-\mu_{P_{1} V}^{\circ}=1
$$

So, using Table 2 and $\mu_{E P_{1}}=\mu_{E P}$,

$$
\begin{aligned}
\lambda_{P_{1}} & =\lambda_{P 1} \mu_{P_{1} E}-\lambda_{P_{1}} \mu_{P_{1} V}^{\circ} \\
& =\lambda_{E} \mu_{E P_{1}}-\lambda_{V} \mu_{V} \stackrel{\circ}{P_{1}} \\
& =\frac{1}{2} \lambda_{V}\left(\mu_{V E} \mu_{E P}-2 \mu_{V P_{1}}\right) .
\end{aligned}
$$

Therefore,

$$
\begin{aligned}
\nu_{1}(P) & =\frac{\lambda_{P_{1}}}{\lambda_{P}} \\
& =\mu_{P V} \frac{\mu_{V E} \mu_{E P}-2 \mu_{V P_{1}}}{\mu_{V E} \mu_{E P}} \\
& =\mu_{P V}\left(1-\frac{2 \tau}{\mu_{V E} \mu_{E P}}\right),
\end{aligned}
$$

where $\tau:=\mu_{V P_{1}}$, the expected number of plate-side interiors adjacent to a typical vertex. In the prism tessellation, $\tau=4$. Obviously, $v_{0}(P)=v_{1}(P)$.

Every plate $p \in P$ is contained in two cells, $z$ and $z^{\prime}$ say. It lies in a facet of each, say $z_{2}$ of $z$ and $z_{2}^{\prime}$ of $z^{\prime}$. Consider a side $p_{1}$ of $p$, and suppose that there exists a vertex $v \subset \stackrel{\circ}{p_{1}}$. If $v$ is not a hemi-vertex of the tessellation then

(a) $\stackrel{\circ}{p_{1}}$ is contained in a ridge of $z$ and also in a ridge of $z^{\prime}$-as is $v$.

If $v$ is a hemi-vertex then either (a) is true or

(b) $\stackrel{\circ}{p}_{1}$ is contained in a ridge of one of the cells, $z$ or $z^{\prime}$, and in the interior of a facet of the other cell-as is $v$. 


\section{8. $\lambda_{X}, v_{j}(X)$ and $\mu_{X Y}$ in the spatial case}

Using the notation $f$ defined in (6), together with

$$
t(x):=2 \mu_{V E} \mu_{E P}-x\left(\xi \mu_{V E}-2 \kappa\right),
$$

in Tables 5 and 6 we express $v_{j}(X)$, and the remaining $\lambda_{X}$.

We also tabulate, in Table 7, some additional $\mu_{X Y}$ formulae (supplementing Table 2). Tables 2 and 7 contain all $\mu$-values which can be expressed in terms of our three cyclic mean adjacency parameters, $\mu_{V E}, \mu_{E P}$, and $\mu_{P V}$, and our four Greek parameters, $\xi, \kappa, \psi$, and $\tau$.

We refer the reader to Appendix B for proofs of the results in Table 7, and for methods to deal with nonprimitive objects of dimension 0 (which have in their formulae various second moments, or subtype first moments such as $\mu_{X Y}$, where $X=E[\pi]$ ).

It is impractical to present a full $15 \times 15$ table comprising all $\mu_{X Y}$ formulae, many of which are typographically lengthy. We turn instead to examples.

TABLE 5: Intensities for nonprimitives in spatial tessellations, from (11), (14), (17), (18), (21), and (23).

\begin{tabular}{lc}
\hline \multicolumn{1}{c}{$X$} & $\lambda_{X} / \lambda_{V}$ \\
\hline Facets $Z_{2}$ & $\frac{t\left(\mu_{P V}\right)}{2 \mu_{P V}}$ \\
Ridges $Z_{1}$ & $\frac{1}{2}\left(\mu_{V E}\left(\mu_{E P}-\xi\right)-2 \psi\right)$ \\
Apices $Z_{0}$ & $\frac{1}{2} f(2)-\kappa-\psi$ \\
Facet sides $\left(Z_{2}\right)_{1}$ & $\mu_{V E}\left(\mu_{E P}-\xi\right)-2 \psi$ \\
Facet corners $\left(Z_{2}\right)_{0}$ & $\mu_{V E}\left(\mu_{E P}-\xi\right)-2 \psi$ \\
Plate sides $P_{1}$ & $\frac{1}{2}\left(\mu_{V E} \mu_{E P}-2 \tau\right)$ \\
Plate corners $P_{0}$ & $\frac{1}{2}\left(\mu_{V E} \mu_{E P}-2 \tau\right)$ \\
Edge termini $E_{0}$ & $\mu_{V E}$ \\
Ridge termini $\left(Z_{1}\right)_{0}$ & $\mu_{V E}\left(\mu_{E P}-\xi\right)-2 \psi$ \\
Plate-side termini $\left(P_{1}\right)_{0}$ & $\mu_{V E} \mu_{E P}-2 \tau$ \\
Facet-side termini $\left(\left(Z_{2}\right)_{1}\right)_{0}$ & $2\left(\mu_{V E}\left(\mu_{E P}-\xi\right)-2 \psi\right)$ \\
\hline
\end{tabular}

TABLE 6: Values of $v_{j}(X), j \leq 2$, from (22), (20), (12), (15), (16), and (23).

\begin{tabular}{cccc}
\hline$X$ & $v_{0}(X)$ & $v_{1}(X)$ & $v_{2}(X)$ \\
\hline$E$ & 2 & - & - \\
$Z_{1}$ & 2 & - & - \\
$P_{1}$ & 2 & - & - \\
$\left(Z_{2}\right)_{1}$ & 2 & - & - \\
$P$ & $\mu_{P V}\left(1-\frac{2 \tau}{\mu_{V E} \mu_{E P}}\right)$ & $\mu_{P V}\left(1-\frac{2 \tau}{\mu_{V E} \mu_{E P}}\right)$ & - \\
$Z_{2}$ & $2 \frac{\mu_{V E}\left(\mu_{E P}-\xi\right)-2 \psi}{t\left(\mu_{P V}\right)\left(\mu_{P V}\right)^{-1}}$ & $2 \frac{\mu_{V E}\left(\mu_{E P}-\xi\right)-2 \psi}{t\left(\mu_{P V}\right)\left(\mu_{P V}\right)^{-1}}$ & $\xi \frac{\xi \mu_{V E}-2 \kappa}{f\left(\mu_{P V}\right)}$ \\
$Z$ & $\mu_{Z V}-\mu_{P V} \frac{2(\kappa+\psi)}{f\left(\mu_{P V}\right)}$ & $\mu_{Z E}-\mu_{P V} \frac{\xi \mu_{V E}+2 \psi}{f\left(\mu_{P V}\right)}$ \\
\hline
\end{tabular}


TABLE 7: Other values of $\mu_{X Y}$, where either (i) $x \in X \Rightarrow \operatorname{dim}(x)>0$ and $Y \in\{V, E, P, Z\}$ or (ii) $y \in Y \Rightarrow \operatorname{dim}(y)>0$ and $X \in\{V, E, P, Z\}$. A dash is used to signify that an entry cannot be expressed in terms of the entities defined in this paper.

\begin{tabular}{|c|c|c|c|c|}
\hline \multicolumn{5}{|c|}{$\mu_{X Y}$ values } \\
\hline \multirow{2}{*}{$X$} & \multicolumn{4}{|c|}{$Y$} \\
\hline & $V$ & $E$ & $P$ & $Z$ \\
\hline \multirow{2}{*}{$Z_{2}$} & $\kappa+\mu_{V E}\left(\mu_{E P}-\xi\right)$ & $\mu_{V E}\left(2 \mu_{E P}-\xi\right)$ & $2 \mu_{V E} \mu_{E P}$ & \\
\hline & $2 \mu_{P V} \overline{t\left(\mu_{P V}\right)}$ & $\mu_{P V} \overline{t\left(\mu_{P V}\right)}$ & $\overline{t\left(\mu_{P V}\right)}$ & - \\
\hline \multirow{2}{*}{$Z_{1}$} & $2 \underline{\mu_{V E}\left(\mu_{E P}-\xi\right)-\psi}$ & $\mu_{V E}\left(\mu_{E P}-\xi\right)$ & - & 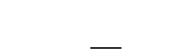 \\
\hline & $\mu_{V E}\left(\mu_{E P}-\xi\right)-2 \psi$ & $\overline{\mu_{V E}\left(\mu_{E P}-\xi\right)-2 \psi}$ & & - \\
\hline \multirow{2}{*}{$\left(Z_{2}\right)_{1}$} & $\mu_{V E}\left(\mu_{E P}-\xi\right)-\psi$ & $\mu_{V E}\left(\mu_{E P}-\xi\right)$ & 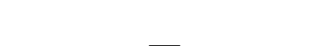 & 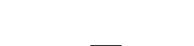 \\
\hline & $\overline{\mu_{V E}\left(\mu_{E P}-\xi\right)-2 \psi}$ & $\overline{\mu_{V E}\left(\mu_{E P}-\xi\right)-2 \psi}$ & - & \\
\hline \multirow{3}{*}{$P_{1}$} & $2 \frac{\mu_{V E} \mu_{E P}-\tau}{2}$ & $\mu_{V E} \mu_{E P}$ & - & - \\
\hline & $\mu_{V E} \mu_{E P}-2 \tau$ & $\mu_{V E} \mu_{E P}-2 \tau$ & & - \\
\hline & $Z_{2}$ & $Z_{1}$ & $\left(Z_{2}\right)_{1}$ & $P_{1}$ \\
\hline$V$ & $\kappa+\mu_{V E}\left(\mu_{E P}-\xi\right)$ & $\mu_{V E}\left(\mu_{E P}-\xi\right)-\psi$ & $2\left(\mu_{V E}\left(\mu_{E P}-\xi\right)-\psi\right)$ & $\mu_{V E} \mu_{E P}-\tau$ \\
\hline E & $2 \mu_{E P}-\xi$ & $\mu_{E P}-\xi$ & $2\left(\mu_{E P}-\xi\right)$ & $\mu_{E P}$ \\
\hline$P$ & 2 & - & - & - \\
\hline$Z$ & - & - & - & - \\
\hline
\end{tabular}

\section{Illustrative examples}

We consider three examples: the spatial STIT model, which provides a three-dimensional version of Figure 2(a); a tetrahedral model; and the prism tessellation illustrated in Figure 4(b).

\subsection{The spatial STIT model}

The $\mu$-values for primitive elements in the spatial STIT model are known (from [23]) and are tabulated below in Table 8(a). Using the values in Table 8(a), we evaluate the terms in Table 6 for the STIT model-see Table 8(b). A list of intensities is compiled in Table 8(c).

It is known that the interior of the typical cell of a spatial STIT model has the same distribution as the interior of the typical cell in a Poisson plane process. So

$$
v_{0}(Z)=8, \quad v_{1}(Z)=12, \quad v_{2}(Z)=6 .
$$

This implies, from the last row of Table 8(b) and the obvious fact from the STIT construction that all edges are $\pi$-edges, that

$$
\psi=2, \quad \kappa=\frac{2}{3}, \quad \text { and } \quad \xi=1 .
$$

These results for $\psi$ and $\kappa$ are new, arising from the theory of this paper. Also new is the result $v_{0}\left(Z_{2}\right)=v_{1}\left(Z_{2}\right)=4$, proved using Table $8(\mathrm{~b})$ and (24).

A spatial STIT tessellation has two types of vertex, illustrated in Figure 5. The vertex type in Figure 5(a), which we call a crossing vertex, is adjacent to two plate-side interiors and four ridge interiors and no facet interior. The vertex type in Figure 5(b) is adjacent to one plate-side interior, one ridge interior, and one facet interior (which makes it a hemi-vertex). Because 
TABLE 8: Results for the spatial STIT process.

\begin{tabular}{|c|c|c|c|c|c|c|c|c|}
\hline \multicolumn{5}{|c|}{ (a) $\mu_{X Y}$ for the primitives } & \multicolumn{4}{|c|}{ (b) $v$ values } \\
\hline \multirow{2}{*}{$X$} & \multicolumn{4}{|c|}{$Y$} & \multirow{2}{*}{$X$} & \multirow{2}{*}{$v_{0}(X)$} & \multirow{2}{*}{$v_{1}(X)$} & \multirow{2}{*}{$v_{2}(X)$} \\
\hline & $V$ & $E$ & $P$ & $Z$ & & & & \\
\hline V & 1 & 4 & 6 & 4 & $P$ & $\frac{6}{7}(6-\tau)$ & $\frac{6}{7}(6-\tau)$ & - \\
\hline E & 2 & 1 & 3 & 3 & $Z_{2}$ & $\frac{6(6-2 \xi-\psi)}{3 \kappa+1}$ & $\frac{6(6-2 \xi-\psi)}{3 \kappa+1}$ & - \\
\hline$P$ & $\frac{36}{7}$ & $\frac{36}{7}$ & 1 & 2 & $Z$ & $24-6(\kappa+\psi)$ & $36-6(2 \xi+\psi)$ & $14-6(2 \xi-\kappa)$ \\
\hline$Z$ & 24 & 36 & 14 & 1 & & & & \\
\hline
\end{tabular}

(c) Intensities

\begin{tabular}{ccccccccccccc}
\hline$X$ & $V$ & $E$ & $P$ & $Z$ & $Z_{2}$ & $Z_{1}$ & $Z_{0}$ & $\left(Z_{2}\right)_{1}$ & $\left(Z_{2}\right)_{0}$ & $P_{1}$ & $P_{0}$ & $X_{0}, \operatorname{dim} X=1$ \\
$\lambda_{X} / \lambda_{V}$ & 1 & 2 & $\frac{7}{6}$ & $\frac{1}{6}$ & 1 & 2 & $\frac{4}{3}$ & 4 & 4 & $\frac{14}{3}$ & $\frac{14}{3}$ & $\lambda_{X_{0}}=2 \lambda_{X}$ \\
\hline
\end{tabular}

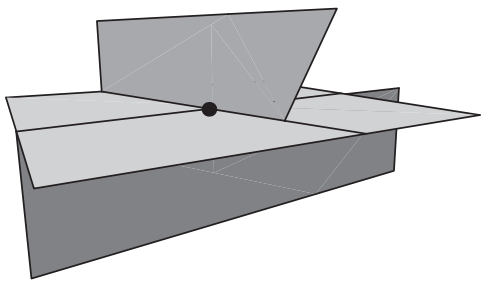

(a)

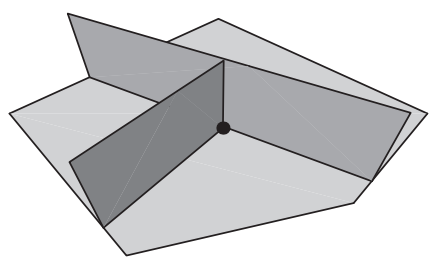

(b)

FIGURE 5: (a) A crossing vertex in the STIT tessellation. (b) A hemi-vertex in the STIT tessellation.

TABLE 9: Various adjacencies for the spatial STIT model.

\begin{tabular}{|c|c|c|c|c|c|c|c|c|c|}
\hline \multicolumn{10}{|c|}{$\mu_{X Y}$ values } \\
\hline \multirow{2}{*}{$X$} & \multicolumn{4}{|c|}{$Y$} & \multirow{2}{*}{$X$} & \multicolumn{4}{|c|}{$Y$} \\
\hline & V & $E$ & $P$ & $Z$ & & $Z_{2}$ & $Z_{1}$ & $\left(Z_{2}\right)_{1}$ & $P_{1}$ \\
\hline$Z_{2}$ & $\frac{26}{3}$ & 10 & $\frac{7}{3}$ & - & V & $\frac{26}{3}$ & 6 & 12 & $\frac{32}{3}$ \\
\hline$Z_{1}$ & 3 & 2 & - & - & $E$ & 5 & 2 & 4 & 3 \\
\hline$\left(Z_{2}\right)_{1}$ & 3 & 2 & - & - & $P$ & 2 & - & - & - \\
\hline$P_{1}$ & $\frac{16}{7}$ & $\frac{9}{7}$ & - & - & $Z$ & - & - & - & - \\
\hline
\end{tabular}

$\kappa=\frac{2}{3}$, the probability that a typical vertex is hemi-type is $\frac{2}{3}$. So $\tau=2 \times \frac{1}{3}+1 \times \frac{2}{3}=\frac{4}{3}$. We check that $\psi=4 \times \frac{1}{3}+1 \times \frac{2}{3}=2$, as derived above.

The first row of Table $8(\mathrm{~b})$ now gives $v_{0}(P)=v_{1}(P)=4$. Interestingly, the typical plate has the same average number of sides as the typical facet. On average, a typical facet comprises $\mu_{Z_{2} P}=\frac{7}{3}$ plates, as follows from a result of Table 7, which we rewrite as Table 9 .

Remark 2. All of the results for this STIT example were unknown prior to this study. Some of the results, however, have been computed in a study (see [29]) conducted in parallel with 
our paper, using only properties of STIT tessellations. The original content in this section is shared with [29]; neither paper has priority over the other.

\subsection{A tetrahedral model}

A spatial tessellation in which all cells are tetrahedra has $v_{0}(Z)=v_{2}(Z)=4$ and $v_{1}(Z)=6$. So two independent equations (in the six unknowns $\mu_{V E}, \mu_{E P}, \mu_{P V}, \xi, \kappa$, and $\psi$ ) can be found from the last row of Table 6, allowing us to express two of the variables in terms of the other four. Choosing $\kappa$ and $\psi$, we have

$$
\begin{gathered}
\kappa=4-\mu_{V E}\left(2-\frac{1}{2} \xi-\frac{\mu_{E P}}{\mu_{P V}}\right) \\
\psi=\mu_{V E}\left(3+\frac{1}{2}\left(\mu_{E P}-\xi\right)-3 \frac{\mu_{E P}}{\mu_{P V}}\right)-6 .
\end{gathered}
$$

Reassuringly, these equations then prove that the expression in Table 6 for $v_{0}\left(Z_{2}\right)$ collapses to 3 , as it should; facets of tetrahedra are triangles.

The best known example of a spatial tessellation comprising only tetrahedral cells is the Poisson-Delaunay tessellation. This is a facet-to-facet tessellation, so $\kappa=\xi=\psi=\tau=0$ and $\mu_{P V}=3$. It is also known, from [15], [18], [19], and [20] (see also [24]), that

$$
\mu_{V E}=2+\frac{48 \pi^{2}}{35} \approx 15.5355 \text { and } \mu_{E P}=\frac{144 \pi^{2}}{24 \pi^{2}+35}=6-\frac{12}{\mu_{V E}} \approx 5.2276
$$

Also,

$$
\lambda_{E}=\left(1+\frac{24 \pi^{2}}{35}\right) \lambda_{V}, \quad \lambda_{P}=\frac{48 \pi^{2}}{35} \lambda_{V}, \quad \lambda_{Z}=\frac{24 \pi^{2}}{35} \lambda_{V} .
$$

The equality in (27) of $\mu_{E P}$ and $6-12 / \mu_{V E}$ is predicted by (25) and (26), one of which becomes redundant in this particular case.

We now construct a tetrahedral model that is not facet-to-facet. We do this by randomly and independently dividing each cell of a Poisson-Delaunay tessellation into two tetrahedral cells. This is achieved by randomly choosing one of the six ridges of the tetrahedral cell (whose vertices we generically label $A B C D$ for discussion purposes, with $A B$ the randomly chosen ridge) and a uniformly random point $Q$ on the opposite ridge $C D$. Construct a dividing plane containing $A B$ and $Q$, an exercise repeated for all cells.

Because each cell divides, the new (denoted by a prime) $\lambda_{Z}$ is

$$
\lambda_{Z}^{\prime}=2 \lambda_{Z}
$$

The new dividing plane $A B Q$ in our generic cell creates two lines: $A Q$ on the original plate $A C D$ and $B Q$ on the original plate $B C D$. Focusing on the original plate $A C D$, we note that it may acquire another line (with one end placed at either $A, C$, or $D$ ) through the division of the other cell adjacent to $A C D$, and this line might cross the line $A Q$ forming a vertex of crossing type (see Figure 5(a)). The original plate $A C D$ will become, post-division, one of the following structures.

- $A C D$ becomes four plates, three being triangular and one four sided, because of the creation of a crossing-type vertex. The new lines have also created four $\pi$-edges. This outcome has probability $\frac{1}{6}$.

- It remains untouched by new lines, with probability $\frac{1}{4}$. 
- Just one of its adjacent cells places a line on $A C D$ (probability $\frac{1}{2}$ ), creating one $\pi$-edge and dividing the plate $A C D$ into two triangular plates.

- Two noncrossing lines are created on $A C D$ (probability $\frac{1}{12}$ ), dividing $A C D$ into three triangular plates and adding two $\pi$-edges to the tessellation.

Each of the new $\pi$-edges mentioned above is adjacent to three plates.

Accounting for these outcomes, we can write (using $P[j]$ to denote the class of plates with $j$ sides)

$$
\begin{aligned}
\lambda_{P}^{\prime} & =\lambda_{Z}+\lambda_{P}\left(\frac{1}{6} \times 4+\frac{1}{4} \times 1+\frac{1}{2} \times 2+\frac{1}{12} \times 3\right)=\frac{128 \pi^{2}}{35} \lambda_{V}, \\
\lambda_{P[4]}^{\prime} & =\frac{1}{6} \lambda_{P}=\frac{8 \pi^{2}}{35} \lambda_{V}, \\
\lambda_{P[3]}^{\prime} & =\lambda_{P}^{\prime}-\lambda_{P[4]}^{\prime}=\frac{120 \pi^{2}}{35} \lambda_{V}, \\
v_{0}^{\prime}(P) & =v_{1}^{\prime}(P)=\frac{3 \lambda_{P[3]}^{\prime}+4 \lambda_{P[4]}^{\prime}}{\lambda_{P}^{\prime}}=\frac{49}{16} \approx 3.0625, \\
\lambda_{E[\pi]}^{\prime} & =\lambda_{P}\left(\frac{1}{6} \times 4+\frac{1}{4} \times 0+\frac{1}{2} \times 1+\frac{1}{12} \times 2\right)=\frac{64 \pi^{2}}{35} \lambda_{V} .
\end{aligned}
$$

Prior to the division, an edge $e$ was adjacent to $m_{P}(e)$ plates and $m_{Z}(e)$ cells; here $m_{P}(e)=$ $m_{Z}(e)$ due to the facet-to-facet property of the Poisson-Delaunay tessellation. After division, $e$ has been split into a random number $R$ of non- $\pi$-edges $e_{1}, e_{2}, \ldots, e_{R}$ by various generic $Q$-points. Edge $e_{i}$ is adjacent post-division to a random number $m_{P}^{\prime}\left(e_{i}\right)$ of plates. Clearly, $\mathrm{E}\left(R \mid m_{Z}(e)\right)=1+\frac{1}{6} m_{Z}(e)$ because each of the $m_{Z}(e)$ cells originally adjacent to $e$ will place its $Q$-point on $e$ with probability $\frac{1}{6}$. Also, $\mathrm{E}\left(m_{P}^{\prime}\left(e_{i}\right) \mid m_{Z}(e), m_{P}(e)\right)=m_{P}(e)+\frac{1}{6} m_{Z}(e)=$ $\frac{7}{6} m_{P}(e)$ for all $i$; this follows because each of these $m_{Z}(e)$ cells will select $e$ as its $A B$-ridge with probability $\frac{1}{6}$. Therefore, using $E[\bar{\pi}]$ to denote the class of non- $\pi$-edges, we have

$$
\begin{aligned}
\lambda_{E[\bar{\pi}]}^{\prime} & =\left(1+\frac{1}{6} \mu_{E Z}\right) \lambda_{E}=\left(1+\frac{1}{6} \mu_{E P}\right) \lambda_{E}=\left(1+\frac{48 \pi^{2}}{35}\right) \lambda_{V}, \\
\lambda_{E}^{\prime} & =\lambda_{E[\pi]}^{\prime}+\lambda_{E[\bar{\pi}]}^{\prime}=\left(1+\frac{16 \pi^{2}}{5}\right) \lambda_{V}, \\
\xi^{\prime} & =\frac{\lambda_{E[\pi]}^{\prime}}{\lambda_{E}^{\prime}}=\frac{64 \pi^{2}}{7\left(5+16 \pi^{2}\right)} \approx 0.5539, \\
\mu_{E P}^{\prime} & =3 \xi^{\prime}+\frac{7}{6} \mu_{E P}\left(1-\xi^{\prime}\right)=\frac{72 \pi^{2}\left(175+176 \pi^{2}\right)}{7\left(5+16 \pi^{2}\right)\left(35+24 \pi^{2}\right)} \approx 4.3824 .
\end{aligned}
$$

Additionally,

$$
\lambda_{V}^{\prime}=\lambda_{V}+\frac{1}{6} \lambda_{P}+\frac{1}{6} \mu_{E Z} \lambda_{E}=\left(1+\frac{32 \pi^{2}}{35}\right) \lambda_{V},
$$

with the three types, original, crossing-type, and $Q$-point vertices, being in proportions 35 : $8 \pi^{2}: 24 \pi^{2}$. The latter two types have $m_{E}(v)=4$, whilst

$$
\mathrm{E}\left(m_{E}(v) \mid v \text { is original }\right)=\mu_{V E}+\frac{1}{2} \mu_{V Z} .
$$


Therefore,

$$
\mu_{V E}^{\prime}=\frac{35 \times\left(\mu_{V E}+\mu_{V Z} / 2\right)+(8+24) \pi^{2} \times 4}{35+32 \pi^{2}}=\frac{14\left(5+16 \pi^{2}\right)}{35+32 \pi^{2}} \approx 6.5012,
$$

using $\mu_{V Z}=\frac{1}{2} f(2)=\frac{1}{2} \mu_{V E} \mu_{E P}-\mu_{V E}+2=96 \pi^{2} / 35$.

Equations (29)-(31) give us three of the seven fundamental parameters; these, combined with the obvious result, $\kappa^{\prime}=0$, enable us to solve (25) for $\mu_{P V}$, yielding

$$
\mu_{P V}^{\prime}=\frac{9\left(175+176 \pi^{2}\right)}{16\left(35+24 \pi^{2}\right)} \approx 3.9560 .
$$

Now solving (26) for $\psi$ yields

$$
\psi^{\prime}=\frac{8 \pi^{2}\left(35+528 \pi^{2}\right)}{\left(35+24 \pi^{2}\right)\left(35+32 \pi^{2}\right)} \approx 4.3429 .
$$

Finally, the seventh and last fundamental parameter, $\tau^{\prime}$, is found from Table 6 combined with (30)-(32) and (28):

$$
\tau^{\prime}=\frac{1}{2} \mu_{V E}^{\prime} \mu_{E P}^{\prime}\left(1-\frac{v_{0}^{\prime}(P)}{\mu_{P V}^{\prime}}\right)=\frac{32 \pi^{2}\left(102 \pi^{2}-35\right)}{\left(35+24 \pi^{2}\right)\left(35+32 \pi^{2}\right)} \approx 3.2176 .
$$

All seven parameters are now in place, $\kappa^{\prime}=0$ and those displayed as (29)-(34).

\subsection{The triangular-prism example (Example 1 continued)}

As this fairly regular tessellation can be visualised clearly in Figure 4(b), the following

\begin{tabular}{|c|c|c|c|c|c|c|c|c|c|c|c|c|c|}
\hline \multicolumn{10}{|c|}{$\mu_{X Y}$ values } & \multicolumn{4}{|c|}{$v$ values } \\
\hline \multirow{2}{*}{$X$} & \multicolumn{9}{|c|}{$Y$} & \multirow{2}{*}{$X$} & \multirow{2}{*}{$\nu_{0}(X)$} & \multirow{2}{*}{$v_{1}(X)$} & \multirow{2}{*}{$v_{2}(X)$} \\
\hline & V & $E$ & & $P$ & $Z$ & $Z_{2}$ & $Z_{1}$ & $\left(Z_{2}\right)_{1}$ & $P_{1}$ & & & & \\
\hline V & 1 & 4 & & 9 & 7 & 16 & 11 & 22 & 14 & $P$ & $\frac{15}{4}$ & $\frac{15}{4}$ & - \\
\hline$E$ & 2 & 1 & & $\frac{9}{2}$ & $\frac{9}{2}$ & $\frac{17}{2}$ & 4 & 8 & $\frac{9}{2}$ & $Z_{2}$ & $\frac{18}{5}$ & $\frac{18}{5}$ & - \\
\hline$P$ & $\frac{27}{4}$ & $\frac{27}{4}$ & & 1 & 2 & 2 & - & - & - & $Z$ & 6 & 9 & 5 \\
\hline$Z$ & 21 & 2 & & 8 & 1 & - & - & - & - & & & & \\
\hline$Z_{2}$ & $\frac{48}{5}$ & $\frac{5}{5}$ & & $\frac{8}{5}$ & - & & & & & & & & \\
\hline$Z_{1}$ & $\frac{11}{3}$ & $\frac{8}{3}$ & & - & - & & & & & & & & \\
\hline$\left(Z_{2}\right)_{1}$ & $\frac{11}{3}$ & $\frac{8}{3}$ & & - & - & & & & & & & & \\
\hline$P_{1}$ & $\frac{14}{5}$ & $\frac{9}{5}$ & & - & - & & & & & & & & \\
\hline \multicolumn{14}{|c|}{ Intensities } \\
\hline \multicolumn{2}{|c|}{$X$} & $E$ & $P$ & $Z$ & $Z_{2}$ & $Z_{1}$ & $Z_{0}$ & $\left(Z_{2}\right)_{1}$ & $\left(Z_{2}\right)_{0}$ & $P_{1}$ & $P_{0}$ & \multicolumn{2}{|c|}{$X_{0}, \operatorname{dim} X=1$} \\
\hline \multicolumn{2}{|c|}{$\lambda_{X} / \lambda_{V}$} & 2 & $\frac{4}{3}$ & $\frac{1}{3}$ & $\frac{5}{3}$ & 3 & 2 & 6 & 6 & 5 & 5 & $\lambda_{X_{0}}=$ & \\
\hline
\end{tabular}
parameters and entries in Table 10 are presented without further comment:

$$
\xi=\frac{1}{2}, \quad \kappa=0, \quad \psi=5, \quad \text { and } \quad \tau=4
$$

TABLE 10: Various parameters in the triangular-prism tessellation. 


\section{Concluding remarks}

For spatial tessellations, we have introduced seven fundamental parameters. These are the three mean adjacencies, $\mu_{V E}, \mu_{E P}$, and $\mu_{P V}$, which are sufficient to describe the more important topological relationships in facet-to-facet tessellations, and our four Greek parameters, $\xi, \kappa, \psi$, and $\tau$, which are needed in other cases. The constraints which apply to these seven parameters are complicated. So we defer the study of the seven-dimensional parameter space to a later paper.

We note that, even in the simpler facet-to-facet case, no study of the constraints operative on $\mu_{V E}, \mu_{E P}$, and $\mu_{P V}$ has yet been published by other authors.

\section{Appendix A. The planar adjacencies in Table 4}

Lemma 1. If a vertex $v \in V$ is adjacent to $m_{E}(v)$ edges and $m_{Z_{1}}^{\circ}(v)$ side interiors, then it is adjacent to

$$
\begin{aligned}
& \left(m_{E}(v)-m_{Z_{1}}^{\circ}(v)\right) \text { corners } \\
& \left(2 m_{E}(v)-m_{Z_{1}}^{\circ}(v)\right) \text { sides } \\
& m_{E}(v) \text { cells } \\
& m_{E}(v) \text { edge termini, } \\
\text { and } & 2\left(m_{E}(v)-m_{Z_{1}}^{\circ}(v)\right) \text { side termini. }
\end{aligned}
$$

Note that $m_{Z_{1}}^{\circ}(v)$ equals either 1 or 0 , the vertex $v$ respectively being a $\pi$-vertex or not.

This obvious lemma immediately gives us $\mu_{V Y}$ for any object class $Y$ in the tessellation, and this leads to $\mu_{Y V}$ via (5). For example, when $Y$ is the class $Z_{1}$ of cell sides,

$$
\mu_{V Z_{1}}=\mathrm{E}_{V} m_{Z_{1}}(v)=\mathrm{E}_{V}\left(2 m_{E}(v)-m_{Z_{1}}^{\circ}(v)\right)=2 \mu_{V E}-\mu_{V Z_{1}}=2 \mu_{V E}-\phi .
$$

Therefore,

$$
\mu_{Z_{1} V}=\frac{\lambda_{V}}{\lambda_{Z_{1}}} \mu_{V Z_{1}}=\frac{2 \mu_{V E}-\phi}{\mu_{V E}-\phi} .
$$

Objects of dimension 0 lie on the vertices of the tessellation. If $X$ is a class of zerodimensional objects, that is, $X \in\left\{V, E_{0}, Z_{0},\left(Z_{1}\right)_{0}\right\}$, its point process can be viewed as a marked point process: a process of points located at the vertices with a mark at a vertex $v$ of $m_{X}(v)$. So it is easily seen that, for any object class $Y$ in the tessellation,

$$
\lambda_{X} \mu_{X Y}=\lambda_{V} \mathrm{E}_{V}\left(m_{X}(v) m_{Y}(v)\right) .
$$

This identity, combined with Lemma 1, provides us with most of the formulae in Table 4. For example, if $X$ is the class $Z_{0}$ of corners and $Y$ is the class $Z_{1}$ of sides,

$$
\begin{aligned}
\mu_{Z_{0} Z_{1}} & =\frac{\lambda_{V}}{\lambda_{Z_{0}}} \mathrm{E}_{V}\left(m_{Z_{0}}(v) m_{Z_{1}}(v)\right) \\
& =\frac{1}{\mu_{V E}-\phi} \mathrm{E}_{V}\left(\left(m_{E}(v)-m_{Z_{1}}^{\circ}(v)\right)\left(2 m_{E}(v)-m_{Z_{1}}^{\circ}(v)\right)\right) \\
& =\frac{1}{\mu_{V E}-\phi} \mathrm{E}_{V}\left(2 m_{E}(v)^{2}-3 m_{E}(v) m_{Z_{1}}^{\circ}(v)+\left(m_{Z_{1}}^{\circ}(v)\right)^{2}\right) \\
& =\frac{2 \mu_{V E}^{(2)}-3 \phi \mu_{V[\pi] E}+\phi}{\mu_{V E}-\phi},
\end{aligned}
$$


using the fact that $\left(m_{Z_{1}}^{\circ}(v)\right)^{2}=m_{Z_{1}}^{\circ}(v)$ (see Lemma 1) and

$$
\begin{aligned}
\mathrm{E}_{V}\left(m_{Z_{1}}^{\circ}(v)\right) & =\phi \mathrm{E}_{V}\left(m_{Z_{1}}^{\circ}(v) \mid m_{Z_{1}}^{\circ}(v)=1\right)=\phi, \\
\mathrm{E}_{V}\left(m_{E}(v) m_{Z_{1}}^{\circ}(v)\right) & =\phi \mathrm{E}_{V}\left(m_{E}(v) m_{Z_{1}}^{\circ}(v) \mid m_{Z_{1}}^{\circ}(v)=1\right) \\
& =\phi \mathrm{E}_{V}\left(m_{E}(v) \mid m_{Z_{1}}^{\circ}(v)=1\right) \\
& =\phi \mu_{V[\pi] E} .
\end{aligned}
$$

The remaining formulae in Table 4 follow either from the use of (5) or by very simple arguments which we leave to the reader.

\section{Appendix B. The spatial adjacencies}

\section{B.1. Objects of dimension 0}

In the spatial case also, objects of dimension 0 lie on the vertices of the tessellation. So the mathematical approach to adjacency relationships such as $\mu_{X Y}$ when the objects belong to $X$ all have dimension 0 follows the same style as in the planar case. In the spatial case, however, Lemma 2 below replaces Lemma 1.

Lemma 2. If a vertex $v$ is adjacent to $m_{E}(v)$ edges, $m_{E[\pi]}(v) \pi$-edges, $m_{P}(v)$ plates, $m_{Z_{2}}^{\circ}(v)$ facet interiors, $m_{Z_{1}}^{\circ}(v)$ ridge interiors, and $m_{P_{1}}^{\circ}(v)$ plate-side interiors, then it is adjacent to

$$
\begin{aligned}
& \left(m_{P}(v)-m_{E}(v)+2\right) \text { cells, } \\
& \left(m_{Z_{2}}^{\circ}(v)+2 m_{P}(v)-m_{E[\pi]}(v)\right) \text { facets, } \\
& \left(2 m_{P}(v)-m_{E[\pi]}(v)-m_{Z_{1}}^{\circ}(v)\right) \text { ridges, } \\
& \left(2 m_{P}(v)-m_{P_{1}}^{\circ}(v)\right) \text { plate sides, } \\
& \left(2 m_{P}(v)-m_{E[\pi]}(v)-2 m_{Z_{1}}^{\circ}(v)\right) \text { ridge termini }, \\
& 2\left(2 m_{P}(v)-m_{E[\pi]}(v)-2 m_{Z_{1}}^{\circ}(v)\right) \text { facet-side termini, } \\
& m_{E}(v) \text { edge termini, } \\
& 2\left(2 m_{P}(v)-m_{E[\pi]}(v)-m_{Z_{1}}^{\circ}(v)\right) \text { facet sides, } \\
& 2\left(m_{P}(v)-m_{P_{1}}^{\circ}(v)\right) \text { plate-side termini, } \\
& \left(m_{P}(v)-m_{P_{1}}^{\circ}(v)\right) \text { plate corners, } \\
& \left(2 m_{P}(v)-m_{E[\pi]}(v)-2 m_{Z_{1}}^{\circ}(v)\right) \text { facet corners, } \\
& \text { and } \quad\left(m_{P}(v)-m_{E}(v)+2-m_{Z_{2}}^{\circ}(v)-m_{Z_{1}}^{\circ}(v)\right) \text { apices. }
\end{aligned}
$$

Note that $m_{Z_{2}}^{\circ}(v)$ equals either 1 or 0 , the vertex respectively being a hemi-vertex or not.

This lemma gives us $\mu_{V Y}$ for any object $Y$ in the tessellation. For example, when $Y$ is a facet side $\left(Z_{2}\right)_{1}$,

$$
\begin{aligned}
\mu_{V\left(Z_{2}\right)_{1}} & =\mathrm{E}_{V}\left(m_{\left(Z_{2}\right)_{1}}(v)\right) \\
& =2 \mathrm{E}_{V}\left(2 m_{P}(v)-m_{E[\pi]}(v)-m_{Z_{1}}^{\circ}(v)\right) \\
& =2\left(2 \mu_{V P}-\mu_{V E[\pi]}-\psi\right) \\
& =2\left(\mu_{V E}\left(\mu_{E P}-\xi\right)-\psi\right) .
\end{aligned}
$$


For $X \in\left\{V, E_{0}, P_{0}, Z_{0},\left(Z_{1}\right)_{0},\left(Z_{2}\right)_{0},\left(P_{1}\right)_{0},\left(\left(Z_{2}\right)_{1}\right)_{0}\right\}$, we can use (35) to establish $\mu_{X Y}$. For example, if $X$ is the class $\left(P_{1}\right)_{0}$ of plate-side termini and $Y$ is the class of $Z_{2}$ facets, then

$$
\begin{aligned}
\mu_{\left(P_{1}\right)_{0} Z_{2}=} & \frac{\lambda_{V}}{\lambda_{\left(P_{1}\right)_{0}}} \mathrm{E}_{V}\left(m_{\left(P_{1}\right)_{0}}(v) m_{Z_{2}}(v)\right) \\
= & \frac{1}{\mu_{V E} \mu_{E P}-2 \tau} \mathrm{E}_{V}\left(2\left(m_{P}(v)-m_{P_{1}}^{\circ}(v)\right)\left(m_{Z_{2}}^{\circ}(v)+2 m_{P}(v)-m_{E[\pi]}(v)\right)\right) \\
= & \frac{2}{\mu_{V E} \mu_{E P}-2 \tau}\left[\mathrm{E}_{V}\left(m_{P}(v) m_{Z_{2}}^{\circ}(v)\right)+2 \mu_{V P}^{(2)}-\mathrm{E}_{V}\left(m_{P}(v) m_{E[\pi]}(v)\right)\right. \\
& \quad-\mathrm{E}_{V}\left(m_{P_{1}}^{\circ}(v) m_{Z_{2}}^{\circ}(v)\right)-2 \mathrm{E}_{V}\left(m_{P_{1}}^{\circ}(v) m_{P}(v)\right) \\
& \left.\quad+\mathrm{E}_{V}\left(m_{P_{1}}^{\circ}(v) m_{E[\pi]}(v)\right)\right] \\
=\frac{2}{\mu_{V E} \mu_{E P}-2 \tau}[ & \quad-\mu_{V[h] P}+2 \mu_{V P}^{(2)}-\mathrm{E}_{V}\left(m_{P}(v) m_{E[\pi]}(v)\right) \\
& \left.\quad-\psi \mu_{V[h] P_{1}}^{\circ}-2 \mathrm{E}_{V}\left(m_{P_{1}}^{\circ}(v) m_{P}(v)\right)+\mathrm{E}_{V}\left(m_{P_{1}}^{\circ}(v) m_{E[\pi]}(v)\right)\right] .
\end{aligned}
$$

We see that expressions can be quite expansive typographically and involve many second moments.

Obviously, all examples of $\mu_{X Y}$ with $Y$ having dimension 0 can be calculated by first finding $\mu_{Y X}$ and then using (5).

\section{B.2. Objects of dimension 1}

The following lemma establishes the adjacencies $\mu_{X Y}$ (and $\mu_{Y X}$ ) in Table 7 , when $Y$ comprises objects of dimension greater than or equal to 1 and $X=E$.

Lemma 3. If an edge $e \in E$ is adjacent to $m_{P}(e)$ plates and $m_{Z_{2}}^{\circ}(e)$ facet interiors, then it is adjacent to

$$
\begin{aligned}
& \left(m_{P}(e)-m_{Z_{2}}^{\circ}(e)\right) \text { ridges, } \\
& \left(2 m_{P}(e)-m_{Z_{2}}^{\circ}(e)\right) \text { facets, } \\
& m_{P}(e) \text { cells, } \\
& m_{P}(e) \text { plate sides, } \\
\text { and } \quad & 2\left(m_{P}(e)-m_{Z_{2}}^{\circ}(e)\right) \text { facet sides. }
\end{aligned}
$$

Note that $m_{Z_{2}}^{\circ}(e)$ equals either 1 or 0 , the edge respectively being a $\pi$-edge or not.

For example, $\mu_{E Z_{2}}=\mathrm{E}_{E}\left(m_{Z_{2}}(e)\right)=\mathrm{E}_{E}\left(2 m_{P}(e)-m_{Z_{2}}^{\circ}(e)\right)=2 \mu_{E P}-\xi$. Then, by using (5),

$$
\mu_{Z_{2} E}=\frac{\lambda_{E}}{\lambda_{Z_{2}}} \mu_{E Z_{2}}=\frac{\mu_{V E} \mu_{P V}}{t\left(\mu_{P V}\right)}\left(2 \mu_{E P}-\xi\right)=\frac{\mu_{P V}}{t\left(\mu_{P V}\right)} \mu_{V E}\left(2 \mu_{E P}-\xi\right) .
$$

\section{B.3. Higher-dimensional objects}

Formulae for $\mu_{X Y}$ when $\operatorname{dim}(Y) \geq \operatorname{dim}(X) \geq 2$ are more difficult to address. They cannot be expressed in terms of the parameters we have introduced, so they lie outside the scope of this paper. 


\section{References}

[1] Arak, T., Clifford, P. and Surgailis, D. (1993). Point-based polygonal models for random graphs. Adv. Appl. Prob. 25, 348-372.

[2] Cowan, R. (1978). The use of ergodic theorems in random geometry. Suppl. Adv. Appl. Prob. 10, 47-57.

[3] Cowan, R. (1980). Properties of ergodic random mosaic processes. Math. Nachr. 97, 89-102.

[4] Cowan, R. (2004). A mosaic of triangular cells formed with sequential splitting rules. In Stochastic Methods and Their Applications (J. Appl. Prob. Spec. Vol. 41A), eds J. Gani and E. Seneta, Applied Probability Trust, Sheffield, pp. 3-15.

[5] Cowan, R. (2010). New classes of random tessellations arising from iterative division of cells. Adv. Appl. Prob. 42, 26-47.

[6] Cowan, R. And Morris, V. B. (1988). Division rules for polygonal cells. J. Theoret. Biol. 131, $33-42$.

[7] Cowan, R. And Tsang, A. K. L. (1994). The falling-leaf mosaic and its equilibrium properties. Adv. Appl. Prob. 26, 54-62.

[8] Grünbaum, B. and Shephard, G. C. (1987). Tilings and Patterns. W. H. Freeman, New York.

[9] Kendall, W. S. And Mecke, J. (1987). The range of mean-value quantities of planar tessellations. J. Appl. Prob. 24, 411-421.

[10] Leistritz, L. AND Zähle, M. (1992). Topological mean value relationships for random cell complexes. Math. Nachr. 155, 57-72.

[11] Mecke, J. (1980). Palm methods for stationary random mosaics. In Combinatorial Principles in Stochastic Geometry, ed. R. V. Ambartzumian, Armenian Academy of Science, Erevan, pp. 124-132.

[12] Mecke, J. (1983). Zufällige Mosaike. In Stochastische Geometrie, Akademie, Berlin, pp. 232-298

[13] Mecke, J. (1984). Parametric representation of mean values for stationary random mosaics. Math. Operationsforch. Statist. Ser. Statist. 15, 437-442.

[14] Mecke, J., Nagel, W. ANd Weiss, V. (2007). Length distributions of edges in planar stationary and isotropic STIT tessellations. Izv. Akad. Nauk Armenii Mat. 42, 39-60. English translation: J. Contemp. Math. Anal. 42, 28-43.

[15] Miles, R. E. (1970). On the homogeneous planar Poisson point process. Math. Biosci. 6, 85-127.

[16] Miles, R. E. (1988). Matschinski's identity and dual random tessellations. J. Microscopy 151, 187-190.

[17] Miles, R. E. ANd Mackisack, M. S. (2002). A large class of random tessellations with the classic Poisson polygon distribution. Forma 17, 1-17.

[18] Møller, J. (1989). Random tessellations in $\mathbb{R}^{d}$. Adv. Appl. Prob. 21, 37-73.

[19] Muche, L. (1996). The Poisson-Voronoi tessellation. II. Edge length distribution functions. Math. Nachr. 178, 271-283.

[20] Muche, L. (1998). The Poisson-Voronoi tessellation. III. Miles' formula. Math. Nachr. 191, 247-267.

[21] Muche, L. (2005). The Poisson-Voronoi tessellation: relationships for edges. Adv. Appl. Prob. 37, $279-296$.

[22] NAGEL, W. AND WeIss, V. (2005). Crack STIT tessellations: characterization of stationary random tessellations stable with respect to iteration. Adv. Appl. Prob. 37, 859-883.

[23] Nagel, W. AND Weiss, V. (2008). Mean values for homogeneous STIT tessellation in 3D. Image Analysis Stereol. 27, 29-37.

[24] OKabe, A., Boots, B., Sugihara, K. And Chiu, S. N. (2000). Spatial Tessellations: Concepts and Applications of Voronoi Diagrams, 2nd edn. John Wiley, Chichester.

[25] RADECKE, W. (1980). Some mean value relations on stationary random mosaics in the space. Math. Nachr. 97, 203-210.

[26] Schneider, R. And WeIL, W. (2008). Stochastic and Integral Geometry. Springer, Berlin.

[27] Stoyan, D. (1986). On generalized planar random tessellations. Math. Nachr. 128, 215-219.

[28] Stoyan, D., Kendall, W. S. And Mecke, J. (1995). Stochastic Geometry and Its Applications, 2nd edn. John Wiley, Chichester.

[29] ThäLE, C. AND Weiss, V. (2010). New mean values for homogeneous spatial tessellations that are stable under iteration. Image Analysis Stereol. 29, 143-157.

[30] Weiss, V. AND ZäHLE, M. (1988). Geometric measures for random curved mosiacs of $\mathbb{R}^{d}$. Math. Nachr. 138, 313-326.

[31] Z̈̈HLE, M. (1988). Random cell complexes and generalised sets. Ann. Prob. 16, 1742-1766. 